\title{
Changes in context-specificity during memory reconsolidation: Selective effects of hippocampal lesions
}

\author{
Gordon Winocur, ${ }^{1,2,3,4,10}$ Paul W. Frankland, ${ }^{5,6,7}$ Melanie Sekeres, ${ }^{5,6}$ Stuart Fogel, $^{8}$ \\ and Morris Moscovitch ${ }^{1,4,9}$ \\ ${ }^{1}$ Rotman Research Institute, Baycrest Centre, Toronto, Ontario M6A 2E1, Canada; ${ }^{2}$ Department of Psychology, Trent University, \\ Peterborough, Ontario K9J 7B8, Canada; ${ }^{3}$ Department of Psychology, University of Toronto, Toronto, Ontario M5T 1 R8, Canada; \\ ${ }^{4}$ Department of Psychiatry, University of Toronto, Toronto, Ontario M5T 1R8, Canada; ${ }^{5}$ Programs in Neurosciences and Mental \\ Health, The Hospital for Sick Children, Toronto, Ontario M5G 1X8, Canada; ${ }^{6}$ Department of Physiology, University of Toronto, \\ Toronto, Ontario M5S 1A8, Canada; ${ }^{7}$ Institute of Medical Science, University of Toronto, Toronto, Ontario M5S 1A8, Canada; \\ ${ }^{8}$ Centre for Neuroscience Studies, Queen's University, Kingston, Ontario K7L 3N6, Canada; ${ }^{9}$ Department of Psychology, Baycrest \\ Centre, Toronto, Ontario M6A 2E1, Canada
}

\begin{abstract}
After acquisition, memories associated with contextual fear conditioning pass through a labile phase, in which they are vulnerable to hippocampal lesions, to a more stable state, via consolidation, in which they engage extrahippocampal structures and are resistant to such disruption. The process is accompanied by changes in the form of the memory from being context-specific to context-general. However, when revived by a reminder, stable memories once again become labile and susceptible to hippocampal disruption, and memory reconsolidation is needed to stabilize them. This study addressed two questions with respect to this reconsolidation phenomenon: (1) How do reminders reinstate a hippocampally dependent memory trace? (2) As the memory changes from a stable to a labile state after a reminder, does its form remain invariant, or does it also change? Using contextual manipulations at retrieval in a test of contextual fear conditioning, we showed that when the fear-conditioning environment served as a reminder, the reinstated memory regained its context specificity and, as a result, became vulnerable again to the effects of hippocampal lesions. By comparison, exposure to a different environment during the reminder session reinstated a version of the original memory that was dependent primarily on general features of the original context and, consequently, was less affected by hippocampal lesions. These findings, which relate loss of reactivated memories after hippocampal destruction (or inactivation) to changes in memory representation, are interpreted as consistent with the transformation hypothesis of memory processing.
\end{abstract}

[Supplemental material is available online at http:// www.learnmem.org.]

The traditional view of memory formation is that, initially, information is encoded and represented in the hippocampus. With the passage of time, those memories become consolidated in extrahippocampal (presumably neocortical) structures, where they are stored and available for retrieval without hippocampal involvement. An important element of this process, termed systems consolidation, is that the long-term memory represented in the neocortex is identical to that initially formed in the hippocampus (Squire and Alvarez 1995; Dudai 2004).

An alternative view states that the shift in memory representation from hippocampal to extrahippocampal structures entails a transformation of the initial memory from one that is highly detailed and context-specific to one that is more schematic, representing only general features of the environment (Moscovitch et al. 2005; Winocur et al. 2007). The transformation view has received support from studies involving contextual fear conditioning in which animals associate an aversive foot shock with the contextual environment and exhibit freezing behavior when placed in that environment (Biedenkapp and Rudy 2007; Wiltgen

${ }^{10}$ Corresponding author.

E-mail gwinocur@rotman-baycrest.on.ca; fax (416) 785-2474. Article is online at http://www.learnmem.org/cgi/doi/10.1101/lm.1447209. and Silva 2007a; Winocur et al. 2007). These investigators showed that at relatively short delays following training, normal animals' memory for the contextual fear response is specific to the training context in which the response was learned. However, at longer delays, the specificity declines and the freezing response generalizes to environments that only slightly resemble the original training environment. The latter, which we refer to as contextgeneral memory, is considered comparable to semantic memory in the human literature (Rosenbaum et al. 2001). The same effect has been observed in an appetitive, socially acquired food preference task (Winocur et al. 2007). While some form of context specificity may be retained over long intervals without the hippocampus, such memories are more fragile and differ in important ways from those that are hippocampally dependent (Wang et al. 2009). A critical element in this theoretical approach is that context-specific and context-general memories, represented as they are in different brain structures, can coexist, although one or the other may dominate under different conditions (for review of converging evidence in support of the transformation view, see Moscovitch et al. 2006).

Issues related to memory representation are also relevant to the reconsolidation phenomenon (Sara 1973; Nader 2003a; Morris et al. 2006), which is the focus of this paper. In reconsolidation, 
a stable memory trace, when revived by a reminder, once again becomes labile and susceptible to amnestic agents. With respect to contextual fear conditioning, Debiec et al. (2002) found that reviving memories that have become independent of the hippocampus returns them to a state in which they are vulnerable to hippocampal disruption (for a consideration of conditions that limit this effect, see Biedenkapp and Rudy [2004]). This phenomenon runs counter to systems consolidation theory, which stipulates that, once consolidated, a memory remains fixed and immutable in neocortex. Nor can this theory explain why a memory should once again be disrupted by treatments that selectively affect the hippocampus if the memory is no longer represented there (Nader 2003a). To deal with these problems, Nader (2003a,b) proposed a systems reconsolidation hypothesis whereby the reminder-induced reactivation process causes the trace again to become hippocampus-dependent and susceptible to hippocampal disruption.

Two important questions arise with respect to the memory representation and reconsolidation: (1) How do reminders reinstate a hippocampus-dependent memory trace? and (2) as the memory changes from a stable to a labile state after a reminder, does its form remain invariant, or does it also change? The transformation hypothesis offers a perspective that addresses both of these questions and shows how they are related. By this view, reminding the animal with the conditioning environment directly affects the representation of the memory such that the contextspecific, hippocampus-dependent memory dominates, whereas before the reminder, the nonhippocampal, context-general memory prevailed. It follows that disrupting hippocampal function will interfere with the expression of the context-specific memory. This account extends the systems reconsolidation hypothesis (Nader 2003a,b) by emphasizing a dynamic relationship between contextspecific and context-general memories across time.
In this study, we used contextual manipulation at retrieval to test the hypothesis that memories undergo transformation after a reminder in a memory reconsolidation paradigm. Rats received contextual fear conditioning in a distinctive context (CXT-A) and $28 \mathrm{~d}$ later were reminded of the training experience by being placed briefly in that same context or in a different context (CXTB) that resembled CXT-A only in its general features. The animals then received hippocampal lesions or control surgery and, upon recovery, their memory for the contextual fear response was tested either in CXT-A or CXT-B. Their performance was compared with that of lesioned and control groups that were similarly tested after a short or long delay, but without a reminder. Following the transformation hypothesis, two critical predictions were made: (1) the memory reactivated by CXT-A, the context-specific reminder, would engage the hippocampus and make the memory vulnerable to hippocampal lesions; and (2) the memory reactivated by CXT-B, the context-general reminder, would be nonspecific, independent of the hippocampus, and, consequently, unaffected by hippocampal lesions.

\section{Results}

\section{Behavioral}

The mean preoperative freezing times for all groups in the $60 \mathrm{sec}$ before and the $60 \mathrm{sec}$ after fear conditioning groups and during the reminder sessions of the Reminder Condition are presented in Supplemental Table 1 . There were no differences between any of the groups on these measures (ANOVA; $P>0.05$ ).

\section{No-Reminder Condition}

Figure 1 presents the results for effects of delay and context change on memory for a learned contextual fear response without reminders.

\section{No Reminder}
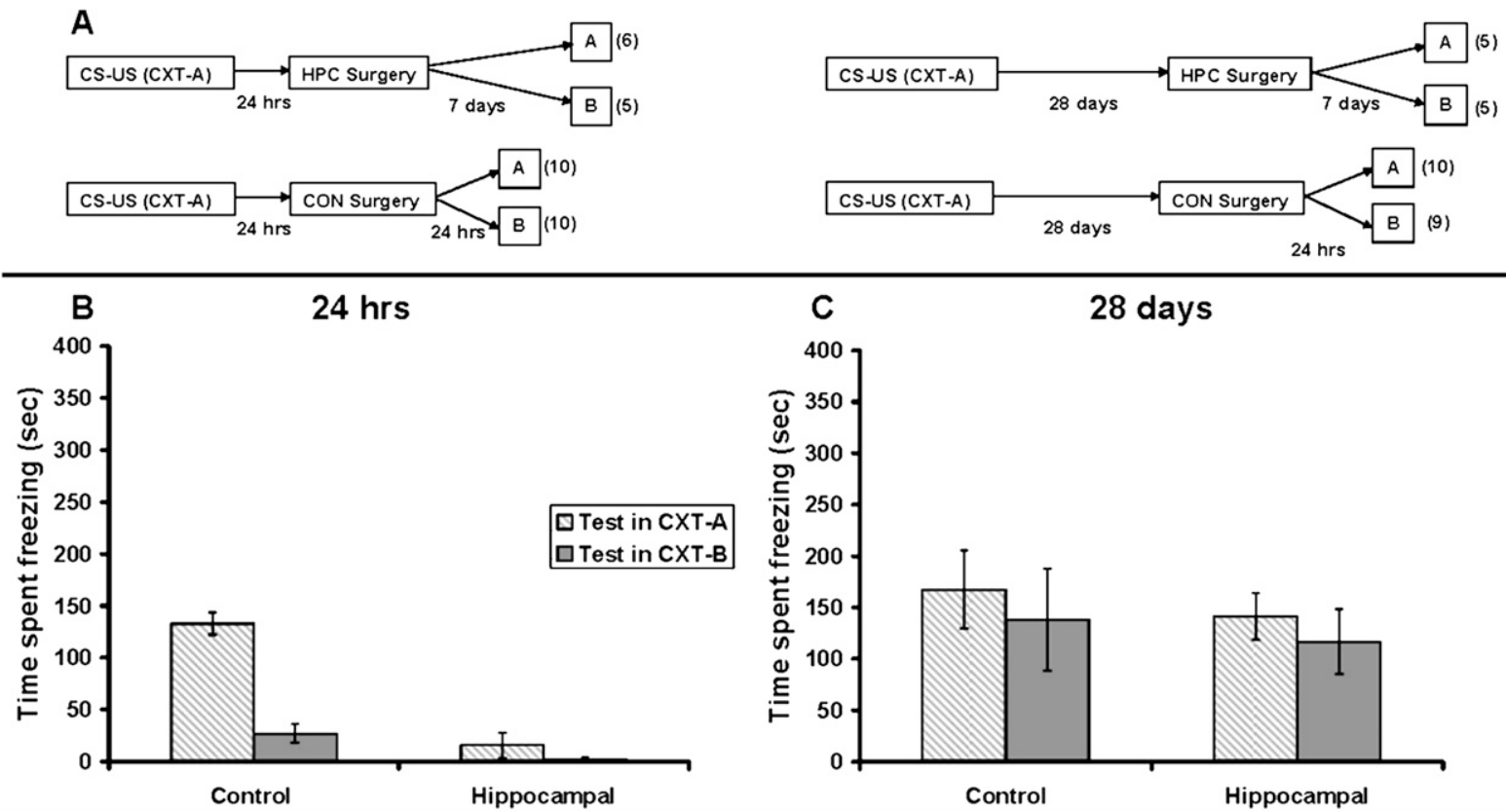

Figure 1. No-Reminder Condition. (A) Schematic representations of timelines for experimental procedures. The numbers in parentheses represent the number of rats in the various groups. $(B, C)$ Contextual fear memory in control and hippocampal groups measured in terms of time spent freezing in the test session after conditioning; surgery intervals of $24 \mathrm{~h}(B)$ and $28 \mathrm{~d}(C)$ were used. In the 24-h test, controls displayed context specificity for the fear memory, while hippocampal rats exhibited no memory of the fear response. At $28 \mathrm{~d}$, both control and hippocampal groups exhibited strong contextual fear memory in the training context (CXT-A) and in a different context (CXT-B) with no context specificity. Error bars represent \pm SEM. 
When tested at the short delay (Fig. 1B), control rats froze much more in the context in which they were trained (CXT-A) than in a different context (CXT-B), but at the long delay (Fig. 1C), they froze equally and extensively in both contexts. This was confirmed by a significant context $\times$ delay interaction, $F_{(1,35)}=6.00$, $P<0.02$, that was due to the reduced freezing by the control group tested at the short delay in CXT-B.

When hippocampal lesions were produced within $24 \mathrm{~h}$ of training, rats exhibited little freezing when tested in either context (Fig. 1B). However, when hippocampal lesions were made $28 \mathrm{~d}$ post-training, when performance was governed by general features, there was no effect and the lesioned rats exhibited considerable freezing in both contexts (Fig. 1C).

\section{Reminder Condition}

In the Reminder Condition, reminders were provided $28 \mathrm{~d}$ after fear conditioning. Providing a CXT-A reminder restored context specificity in control animals, so that they froze much more when subsequently tested in CXT-A than in CXT-B (Fig. $2 \mathrm{~A} ; t_{(18)}=3.42$, $P<0.005)$. This contrasts with the considerable freezing exhibited by controls in both contexts when tested at the same long delay without a reminder. ANOVA confirmed a significant reminder $X$ context interaction, $F_{(1,35)}=4.02, P=0.05$, that was due to reduced freezing by the control group that was reminded in CXT-A and tested in CXT-B. As indicated above, this was the same pattern exhibited by control rats tested shortly after acquisition (Fig. 1A).

Providing a CXT-B reminder led to comparable freezing by controls in both contexts (Fig. $2 \mathrm{C} ; t_{(28)}=1.91, P>0.05$ ), indicating that CXT-B was not as effective in restoring context specificity as CXT-A. The latter results parallel the lack of context specificity exhibited by control rats tested at the long delay in the No-
Reminder Condition (Fig. 1C). In the latter condition, control rats froze equally in CXT-A and CXT-B $(t<1)$.

In absolute terms, control rats tested in CXT-B exhibited comparable levels of freezing with reminders (Fig. 2B,C) as without reminders (Fig. 1B,C) regardless of delay. By comparison, controls tested in CXT-A after either a CXT-A or CXT-B reminder froze approximately twice as much as controls tested in CXT-A without a reminder, at both short and long delays.

In the reconsolidation test, hippocampal lesions after a CXTA reminder virtually eliminated freezing (Fig. 2B). After a CXT-B reminder, when compared with controls that also received a CXT$B$ reminder, rats with hippocampal lesions (HPC group) exhibited reduced freezing when tested in CXT-A $\left(t_{(22)}=3.57, P<0.002\right.$; Fig. 2C) but not to the level seen after a CXT-A reminder (Fig. 2B). The lesions, however, had no effect on freezing in CXT-B $(t<1)$ after a CXT-B reminder (Fig. 2C). These results indicate that the effectiveness of hippocampal lesions is related to the specificity of the memory that is reinstated by the reminder. These results are consistent with the findings, in the No-Reminder Condition, that hippocampal lesions made $24 \mathrm{~h}$ after training, when memory was context specific, abolished freezing, but hippocampal lesions made $28 \mathrm{~d}$ after training, when memory was context general, had no effect (Fig. 1B,C).

Clearly, the two reminders in the reconsolidation tests affected the HPC and control groups differently. The effects of the CXT-B reminder are of interest because they were in opposite directions. As noted in Figure 2C, after a CXT-B reminder, control rats froze slightly more when tested in CXT-A than in CXT-B, whereas the reverse was seen in the HPC group. This finding, which was confirmed by a significant group $\times$ test environment interaction $\left(F_{(1,43)}=4.54, P<0.05\right)$, is discussed below.

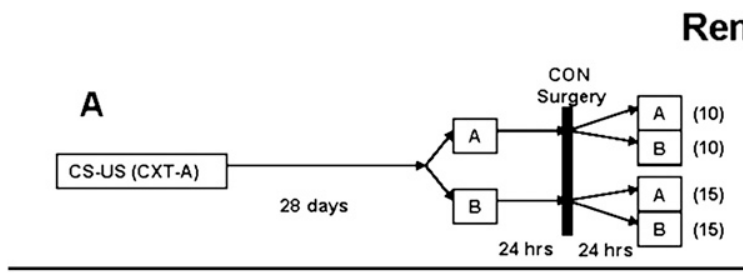

\section{Reminder}
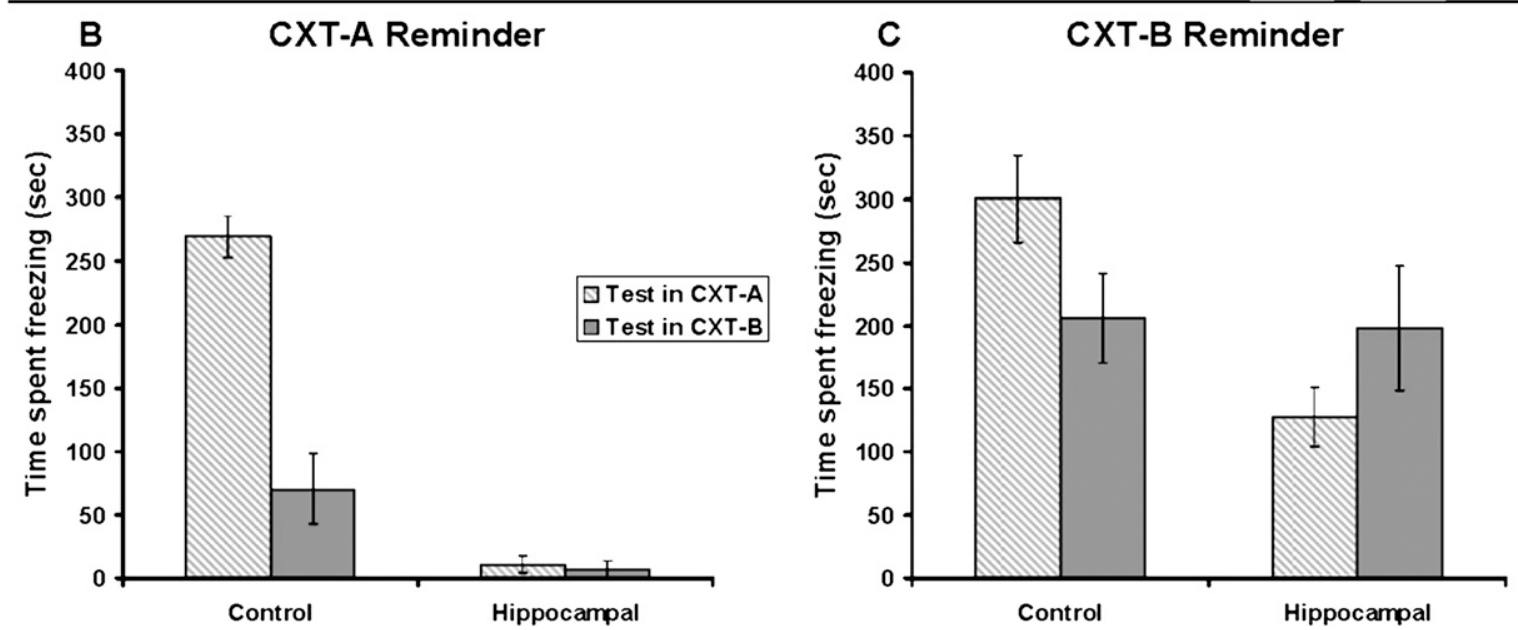

Figure 2. Reminder Condition. $(A)$ Schematic representations of timelines for experimental procedures. The numbers in parentheses represent the number of rats in the various groups. $(B, C)$ Contextual fear memory in control and hippocampal groups when either CXT-A (B) or CXT-B (C) was provided as a reminder $28 \mathrm{~d}$ after fear conditioning in CXT-A and $24 \mathrm{~h}$ before surgery. CXT-A, as a reminder, restored context specificity of the contextual fear memory and, consequently, controls froze only when tested in CXT-A. Hippocampal lesions eliminated the context-specific memory when it was reactivated by CXT-A. CXT-B was not an effective reminder for context specificity and resulted in generalized freezing in control and HPC groups. Error bars represent \pm SEM. 
Finally, the time spent freezing in each minute of the 8-min test periods was examined to determine whether HPC and control groups exhibited different patterns of discrimination or generalization over the test periods. In the No-Reminder Condition, the data were analyzed by comparing the time spent freezing by HPC and control groups in the first and second 4-min time periods when tested in CXT-A or CXT-B at the short and long delays. In general, freezing times were distributed equally over the two time periods, and ANOVA revealed no significant interactions or main effects involving time period (all $P$ s $>0.05$ ). Similar comparisons, performed on the data of the Reminder Condition, yielded no effect of time period (all Ps > 0.05). Thus, the evidence indicates that the relative influence of context-specific and context-general memories was fairly consistent over the entire test period in the various conditions. (Time spent freezing on a minute-by-minute basis is provided for all conditions in Supplemental Table 1.) ${ }^{11}$

\section{Histological evaluation}

Figure 3A provides photomicrographs of coronal sections of a representative hippocampal lesion (left) in relation to a normal brain (right) and Figure 3B shows schematic drawings of minimal (gray) and maximal (black) extents of lesions meeting our histological criteria.

The nature and extent of lesions were similar to those reported in recent studies (Winocur et al. 2005, 2007). In all the rats with hippocampal lesions, damage extended bilaterally to dorsal and ventral regions of the structure. Half the lesioned rats sustained damage to $50 \%-80 \%$ of the hippocampus proper. Approximately $25 \%$ had very large lesions that affected $80 \%-$ $100 \%$ of the hippocampus, including extensive damage to all the subfields (CA1-CA3, dentate gyrus). Five rats with lesions that affected $30 \%-40 \%$ of the hippocampus were included because their performance fell within the range of their respective group. Overall, the median value for hippocampal destruction was $72 \%$, with the extent and pattern of damage to dorsal and ventral regions similar in the No-Reminder and Reminder Conditions. In all cases, extrahippocampal damage was minor or nonexistent.

ANOVA, performed on the measures of total hippocampal lesion, confirmed that there was no difference in the average volume of hippocampal damage across the eight independent HPC groups in this study $(F<1)$. As well, there was no evidence that lesion size was related to the amount of freezing in the NoReminder $(r=0.23, P<0.32)$ and Reminder $(r=0.18, P<0.74)$ Conditions.

\section{Discussion}

These results confirm a number of established findings in the literature. For example, we found temporally graded retrograde amnesia for contextual fear conditioning following hippocampal lesioning, with recent memories being far more impaired than remote memories, which were relatively preserved (Kim and Fanselow 1992; Anagnostaras et al. 1999; Winocur et al. 2007; but see Lehmann et al. 2007; Sutherland et al. 2008 for reports of conditions in which extensive retrograde amnesia of a contextual fear response after hippocampal lesioning can occur). Although we had to allow seven days' recovery time after hippocampal surgery, this result is consistent with those obtained in studies using protein synthesis inhibitors and shorter delays between reactivation and testing (e.g., Milekic and Alberini 2002; for other studies, see the review by Nader and Hardt 2009). This indicates that the time of surgery, and not the necessary delay imposed by recovery from surgery, was the determining factor in our results.

\footnotetext{
${ }^{11}$ An anonymous reviewer suggested this analysis.
}

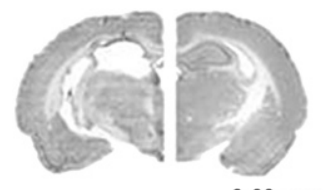

$3.30 \mathrm{~mm}$

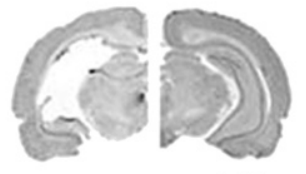

$5.20 \mathrm{~mm}$

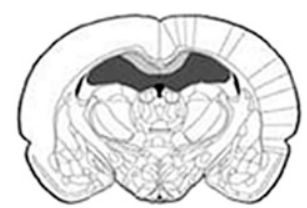

$3.30 \mathrm{~mm}$

$.5 .20 \mathrm{~mm}$

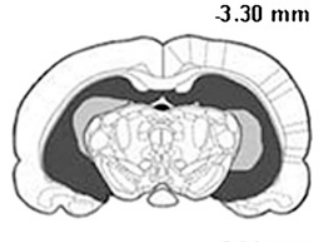

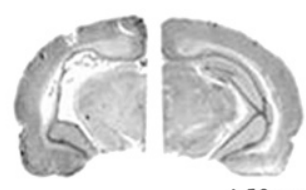

$4.50 \mathrm{~mm}$
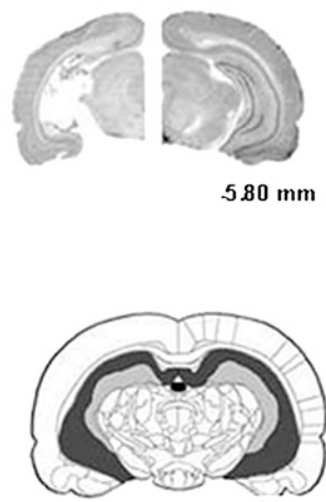

$4.80 \mathrm{~mm}$

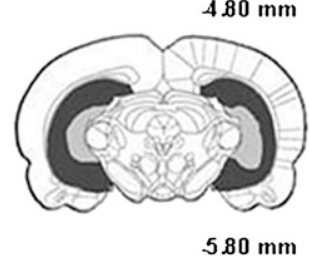

Figure 3. Lesion representations. (Top) Photomicrographs of representative hippocampal lesion (left) in relation to a normal brain (right). (Bottom) Schematic drawings of minimal (gray) and maximal (black) extents of hippocampal lesions meeting the histological criterion included in our experiment.

We also obtained further evidence for the contextual specificity of recently acquired fear responses in the No-Reminder Condition (Biedenkapp and Rudy 2007; Wiltgen and Silva 2007a,b; Winocur et al. 2007). When control animals were tested one day after training, they showed freezing only in the context in which they were trained, but when tested at $28 \mathrm{~d}$, the response generalized to other contexts. Taken together, these findings indicate that hippocampal lesions selectively affect context-specific, but not context-general, memories.

With this as background, we now can interpret the major new findings in this study, which address questions concerning changes in memory representation in reconsolidation. Debiec et al. (2002) reported that long-term memories that are resistant to the effects of hippocampal inactivation or lesions become vulnerable once again after a reminder (see also reviews by Nader 2003a; Sara 2007; Nader and Hardt 2009). In this study, we show that this effect is found only for reminders that restore the contextual specificity of the memory. When the original context (CXT-A) serves as a reminder of a learned contextual fear response, a normal rat will show extensive freezing in that context but not in a different context (CXT-B). By comparison, a new context (CXTB) reminder, while capable of activating a fear response to the general context, is not as effective at reinstating context specificity. As can be seen in Figure 2C, although control rats froze in both environments, they froze only slightly more in the original training environment than in the new one.

A second major finding is that the context-specificity of the memory determines its susceptibility to hippocampal lesions. This was seen both in the No-Reminder Condition, after initial acquisition (see Fig. 1B), and in the Reminder Condition, when rats were tested following a reminder with the conditioning context 
(CXT-A) $28 \mathrm{~d}$ after training (see Fig. 2B). By comparison, CXT-B, as a reminder, effectively reinstated the freezing response in rats with hippocampal lesions. As can be seen in Figure 2C, the HPC groups froze substantially more when tested in CXT-A and CXT-B, following a CXT-B reminder, than they did following a CXT-A reminder (Fig. 2B). Interestingly, whereas control rats froze slightly more in CXT-A than in CXT-B, the HPC groups showed the opposite pattern. This result suggests that the CXT-B reminder revived some components of the context-specific memory but, as would be expected, to a greater degree in control rats.

Taken together the results reveal a feature of the dynamics of memory representation that has interesting theoretical implications. According to the transformation hypothesis, in systems consolidation, a memory undergoes qualitative changes from context-specific to context-general as it becomes represented in a distributed extrahippocampal network. It is now clear from the findings of the Reminder Condition that a similar process occurs in reverse in reconsolidation following a context-specific reminder.

Previous studies examined the effect of intrahippocampal infusion of the protein synthesis inhibitor, anisomycin, on a reactivated contextual fear memory at short and long delays after fear conditioning (Debiec et al. 2002; Frankland et al. 2006). In all cases, the fear conditioning trial, the reminder experience, and testing were conducted in the same context. In line with our results, the consistent finding was that hippocampal disruption abolished retrieval of the contextual fear response when the delay between conditioning and reactivation was relatively short. At long delays, comparable to ours, Debiec et al. (2002) found that hippocampal disruption was equally effective in disrupting reactivated recent and remote contextual fear memories. In contrast, at long delays, Milekic and Alberini (2002), Suzuki et al. (2004), and Frankland et al. (2006) found that anisomycin, infused directly into the hippocampus or injected systemically, had no effect, as animals displayed the typical freezing response when replaced in the conditioning chamber. Apart from the fact that these studies used anisomycin to disrupt hippocampal function and we made lesions, there are other procedural differences as well. For example, Frankland et al. (2006) and Suzuki et al. (2004) tested mice in their research, while we, Milekic and Alberini (2002), and Debiec et al. (2002) tested rats. As well, Milekic and Alberini (2002) used an inhibitory avoidance task rather than the more typical contextual fear conditioning. It is difficult to say how such differences affected the results, but if they contributed to differences in the strength of the original memory trace that may be a potential factor (Alberini 2007). It is significant that Frankland et al. (2006) found that systemic infusions of anisomycin, which likely affect both the hippocampus and neocortex, disrupted contextual fear memory at long delays. This suggests, in line with our hypothesis, that when the memory is distributed across both hippocampus and cortex, damage to both regions, but not to either alone, is necessary to disrupt the memory.

Our results go further and suggest that the effect of the CXT-A reminder was to revive the context-specific memory and to reassert its dominance. Because the context-specific memory is hippocampus-dependent, hippocampal lesions destroyed that memory and eliminated freezing. In contrast, the CXT-B reminder reasserted the context-general memory, which was expressed by freezing in both contexts and, consequently, was relatively invulnerable to hippocampal lesions. These findings extend the systems reconsolidation hypothesis (Nader 2003a,b; Nader and Hardt 2009) by relating loss of reactivated memories following hippocampal inactivation or destruction to changes in memory representation; only those reminders that reinstate context specificity are susceptible to such loss. (In a study involving secondorder fear conditioning, Debiec et al. [2006] also considered the effect of reminders on different memories that bear a relationship to each other. Although their study differed from the present one in several ways [e.g., task, amnestic agent, brain region of interest: amygdala], an important similarity is that the ability of an amnestic treatment to disrupt expression of a reactivated memory was related to the nature of the memory and its representation.)

A central assumption of the transformation hypothesis is that a memory undergoes qualitative changes from context-specific to context-general, as the representation shifts from the hippocampus to a distributed extrahippocampal network in systems consolidation (Winocur et al. 2007; see also Moscovitch et al. 2005; Wiltgen and Silva 2007a,b). As we noted above, the reverse process occurs in reconsolidation following a reminder that is closely associated with the environment in which original learning occurred. Importantly, a reminder that is associated with general features of the conditioning environment is also capable of reactivating the learned response, although that memory lacks the context specificity of a newly formed memory.

While the hippocampus clearly mediates context-specific memory, more research is needed to determine which extrahippocampal structures and pathways are implicated in contextgeneral memory. Converging evidence from studies using immediate early gene mapping (Frankland et al. 2004; Maviel et al. 2004; Ross and Eichenbaum 2006), protein synthesis inhibitors (Nader et al. 2000; Morris et al. 2006), temporary region-specific neuronal inactivation (Frankland et al. 2004; Maviel et al. 2004), and lesions (Remondes and Schuman 2004) have identified a number of structures that are involved in the process of long-term memory systems consolidation, with the anterior cingulate cortex as the most prominent candidate (Bontempi et al. 1999; Frankland et al. 2004; Maviel et al. 2004; Frankland and Bontempi 2005). According to our view, these structures would be implicated only in context-general memory, but, to our knowledge, no published study has tested this prediction.

Having established that two types of memory must be taken into account in studies of memory reconsolidation, new questions are raised about the relationship between context-specific and context-general memories and the structures that mediate them. For example, if as shown, the context-general memory is represented in extrahippocampal structures after a long delay (Fig. 1B), why, in the Reminder Condition, did the CXT-A reminder activate the context-specific memory and render it vulnerable to hippocampal lesions, and why was the context-general memory not expressed in the HPC group that had received the CXT-A reminder? These are important questions, and gene expression and imaging techniques should prove useful in finally resolving them (Frankland et al. 2004). One possibility, of course, is that both forms of the memory were eliminated by hippocampal lesions. An alternative suggestion, consistent with our hypothesis that both memories can coexist, is that, over the 24 -h period between the reminder and surgery, during which the context-specific, hippocampus-dependent memory becomes dominant once again, neocortically mediated context-general memories are inhibited or "overshadowed" (Frankland et al. 1998; Anagnostaras et al. 1999; Eisenberg et al. 2003). This inhibitory process may be considered analogous to the supremacy of hippocampal memories vis-à-vis memories mediated by other neural systems (e.g., Packard and McGaugh 1992; Sutherland et al. 2006).

In considering the mechanism of such an inhibitory effect, one possibility is that the specific reminder of the hippocampal memory (i.e., re-exposure to CXT-A) initiates a retrieval process that, once enacted, blocks access to the general memory for a period of time, even if the specific memory is no longer available. Although the inhibitory or retrieval effects outlast the removal of the hippocampus, it is entirely possible that they are not permanent and that the context-general memory may recover with time. The evidence for recovery of contextual fear conditioning is mixed 
(see Nader and Hardt 2009). One prediction that emerges from our data, but which has not been tested, is that if memory does recover, it should be context general.

Another possible mechanism is suggested by the distinction that can be made between a potential memory and an actualized one. The actualization of a memory depends on the presence of appropriate cues that enable the reconstruction and ultimate retrieval of that memory. In our study, following the CXT-A reminder and the actualization of the context-specific memory, the context-general memory, while available in principle, was not accessed directly, did not become actualized, and remained a potential memory. The effect of removing the hippocampus after the CXT-A reminder was to eliminate the only memory that was actualized to that point, leaving the other memory in a dormant state. This interpretation can account for the poor memory exhibited by rats with hippocampal lesions in both contexts (see Fig. 2B). When a CXT-B reminder was given prior to hippocampal destruction, the general memory did become actualized and the hippocampal lesion was much less effective in eliminating it (see Fig. 2C). This interpretation differs from the previous inhibitory interpretation in that no active mechanism is needed to suppress the context-general memory. It merely remains dormant, never having been activated. These ideas are being evaluated in current experiments designed to test specific predictions that follow from the two interpretations.

By focusing on the interplay between memory and its reminders, the transformation hypothesis reinterprets reconsolidation as an instance of memory retrieval. According to this interpretation, the reminders serve as cues to retrieve a previously forgotten or inaccessible context-specific memory. Once retrieved, this memory is again vulnerable to hippocampal disruption. Within this framework, the reminder (reconsolidation) paradigm affords the opportunity to study selectively the neurobiological mechanisms underlying retrieval, in contrast to consolidation paradigms that emphasize memory formation and retention (Dudai 2006). Viewed in this way, the study of consolidation and reconsolidation processes has much to contribute to our understanding of the relation between encoding and retrieval in learning and memory.

\section{Materials and Methods}

\section{Subjects}

Male, adult Long-Evans rats, $\sim 5$ mo old, were obtained from the Charles River Laboratories (Saint-Constant, Québec, Canada) and served as subjects in both experiments. The rats were housed individually in shoebox cages with unlimited access to standard laboratory chow and water and maintained on a reversed 12-h light/dark cycle (lights on at $1800 \mathrm{~h}$ and off at $0600 \mathrm{~h}$ ). All testing took place during the high activity, dark phase of the cycle.

The experimental protocol and all handling procedures conformed to guidelines set out by the Canadian Council on Animal Care and were approved by the Trent University Animal Care Committee. Throughout the research, the rats were examined regularly by a veterinarian.

\section{Apparatus}

All rats received fear conditioning in a chamber $(50 \times 40 \times 18 \mathrm{~cm})$ that consisted of four walls made of clear Plexiglas, a hinged clear Plexiglas roof with holes to allow ventilation, and a floor that consisted of metal rods, spaced $1.3 \mathrm{~cm}$ apart. As part of the fear conditioning procedure, a tone, presented through a centrally mounted speaker attached to the roof of the box, was paired with a $1.5-\mathrm{mA}$ foot shock that was delivered by a TechServe Model $452 \mathrm{~A}$ shock generator.

The fear-conditioning chamber was positioned on a table, $1.3 \mathrm{~m}$ above the floor, and situated in the center of a large standard laboratory room $(6.3 \times 6.1 \mathrm{~m})$. The room contained standard furniture (e.g., desk, table, bookshelf along one wall, etc.), as well as pictures, light fixtures, etc., on the walls. Illumination was provided by overhead fluorescent lights under rheostatic control. The environment in which rats received fear conditioning is referred to as Context A (CXT-A).

In both the Reminder and No-Reminder Conditions, approximately half the rats were tested in a different environment (CXTB). The test chamber used in CXT-B was smaller $(40 \times 30 \times 18 \mathrm{~cm})$ than the CXT-A chamber. It was also made of Plexiglas but with semi-transparent tinted walls. The roof was clear Plexiglas with ventilation holes, and the floor consisted of metal rods, spaced $1.3 \mathrm{~cm}$ apart. The CXT-B chamber was placed in a smaller room $(3.4 \times 2.1 \mathrm{~m})$ on a table that was situated against a wall. Care was taken to ensure that the configuration of furniture, pictures, lighting conditions, etc., were different from that of the room in which fear conditioning took place. Thus, CXT-B resembled CXT-A only in terms of the latter's general features-that is, a Plexiglas test chamber placed in a room other than its living environment.

\section{Fear conditioning procedure}

This procedure, which was the same for all conditions, was adapted from Anagnostaras et al. (1999) and identical to that routinely followed in our laboratory (e.g., Moses et al. 2007; Winocur et al. 2007). Each rat received one fear conditioning trial that began with the rat being placed in the chamber in CXT-A and allowed to explore freely for $5 \mathrm{~min}$. The rat then received 10 toneshock pairings at variable intervals. Beginning $30 \mathrm{sec}$ after the last shock and over a 64-sec period, freezing behavior was recorded every $8 \mathrm{sec}$ (eight observations). Following Anagnostaras et al. (1999), freezing was defined by an immobilized crouching response in which the only detectable movement was the rat's breathing. The rat was then removed from the box and returned to its home cage. The test session was video recorded, and the time spent freezing was manually recorded using a stopwatch.

\section{Testing procedures}

Testing procedures were identical, regardless of whether rats were tested in CXT-A or CXT-B, and whether or not they had received a pretest reminder session. Testing consisted of a single trial in which the rat was placed in the appropriate chamber for $8 \mathrm{~min}$ and, in the absence of the tone, the amount of time spent freezing was recorded. Immediately after testing, the rat was removed from the chamber and returned to its home cage. As in the conditioning session, the test session was video recorded and the time spent freezing was recorded.

In repeated experiments, we found these procedures to be highly effective in producing a strong contextual fear response in normal rats (Winocur et al. 2006, 2007; Moses et al. 2007). During testing, we and others (e.g., Phillips and Ledoux 1994) consistently find that rats with hippocampal lesions and control rats establish a conditioned freezing response to the tone equally well. When tested subsequently in the same context, but without the tone, the equally consistent finding is that only the control rats continue to freeze. In the study by Phillips and Ledoux (1994), the effects of pairing a tone with foot shock on contextual fear conditioning was compared directly with the effects of presenting the foot shock alone. The results showed that lesions to the hippocampus were far more effective in abolishing the contextual fear response in the paired than in the unpaired condition. Since freezing in response to the tone, was not of interest in this study, it was not measured.

\section{Surgical and histological procedures}

Rats were anesthetized with isoflurane respiratory anesthetic. Stereotaxic coordinates were based on the Paxinos and Watson (1997) atlas and located in relation to bregma and the horizontal skull surface. The procedure for making hippocampal lesions was slightly modified from the technique developed by Jarrard and Meldrum (1993). Using a small dental burr, eight holes were drilled through the skull directly above the hippocampus in each 
hemisphere. Hippocampal lesions were produced by 10 intracranial microinjections of a solution containing the cellular neuro-

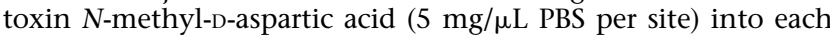
hemisphere. The injection coordinates were calculated from a level head with respect to bregma: $3.1 \mathrm{~mm}$ posterior (p), $\pm 1 \mathrm{~mm}$ lateral (l), and $3.6 \mathrm{~mm}$ ventral (v); 3.1 (p), 2 (l), $3.6(\mathrm{v}) ; 4.1$ (p), 2 (l), 4 (v); 4.1 (p), $3.5(\mathrm{l}), 4$ (v); 5 (p), 3 (l), $4.1(\mathrm{v}) ; 5$ (p), $5.2(\mathrm{l}), 5(\mathrm{v}) ; 5$ (p), 5.2 (l), $7.3(\mathrm{v}) ; 5.8(\mathrm{p}), 4.4(\mathrm{l}), 4.4(\mathrm{v}) ; 5.8(\mathrm{p}), 5.1(\mathrm{l}), 6.2(\mathrm{v}) ; 5.8(\mathrm{p}), 5.1$ (l), $7.5(\mathrm{v})$. The solution was infused at a rate of $0.4 \mu \mathrm{L} / \mathrm{min}$ through 30-gauge stainless steel needles for $38 \mathrm{sec}$, using a $10-\mu \mathrm{L}$ syringe attached to a motorized infusion pump. The last two ventral hippocampal sites were injected for $2 \mathrm{~min}$ each. The needles were removed 2 min after each injection. In the control procedure, incisions and burr holes were identical to the lesioned animals with the exception that there was no penetration of brain tissue. To facilitate recovery from surgery, all rats were given intraperitoneal injections of diazepam $(10 \mathrm{mg} / \mathrm{kg})$.

At the end of the experiment, rats with hippocampal lesions were deeply anesthetized with sodium pentobarbital $(65 \mathrm{mg} / \mathrm{kg})$ and perfused intracardially with $0.9 \%$ saline followed by $10 \%$ formalin. The fixed brains were removed from the skull and stored in $10 \%$ formalin. Brains were transferred to a $30 \%$ buffered sucrose solution $48 \mathrm{~h}$ prior to sectioning. The brains were then frozen and sliced at $40 \mu \mathrm{m}$, using a cryostat. Every fifth section was mounted on a gel-coated slide and stained with neural red.

Lesion size was estimated for each rat by determining the percent of hippocampal tissue damaged within a cross-section of brain slices corresponding to Figures 28, 34, 37, and 40 in Paxinos and Watson (1997). Sections were examined under a light microscope, and the area of tissue damaged was manually recorded on templates of the hippocampus for the four figures, corresponding to $-3.30,-4.50,-5.20,-5.80 \mathrm{~mm}$ from bregma. The volume of destroyed hippocampal tissue was determined as a percentage by dividing the lesioned area by the total area of hippocampal tissue for the appropriate figure. For each section, the percentage of hippocampal tissue damaged in the dorsal and ventral regions was calculated for both the left and right hippocampus. An estimate of the total hippocampal lesion was also calculated for each brain.

\section{No-Reminder Condition}

Sixty rats were used in this condition. Of these, 21 rats received hippocampal lesions and 39 received control lesions. Fear conditioning was conducted in CXT-A as described above. Hippocampal or control surgery was performed $24 \mathrm{~h}$ or $28 \mathrm{~d}$ after the fear conditioning trial. Rats in the control groups were tested the next day in either CXT-A or CXT-B. Rats in the HPC groups were tested either in CXT-A or CXT-B $7 \mathrm{~d}$ after surgery. A schematic outline of the procedure for the No-Reminder Condition and the number of rats in each group are provided in Figure 1.

\section{Reminder Condition}

Eighty rats were used in this condition. Of these, 30 rats received hippocampal lesions and 50 rats received control surgery. Initially, all rats received a single fear conditioning session in CXT-A, as described above. Twenty-eight days later, each rat received a reminder session in which it was transported to the appropriate test room and placed either in the chamber of CXT-A or in the chamber of CXT-B. Neither the tone nor foot shock was presented. After the 60-sec reminder session, the rat was then returned to its home cage.

One day after the reminder session, hippocampal lesion or control surgery was performed. Twenty-four hours later, memory for the contextual fear response was tested in the control rats either in the chamber of CXT-A or the chamber of CXT-B, according to the test procedures described above. Rats with hippocampal lesions were tested seven days later in either CXT-A or CXT-B.

A schematic outline of the procedure for the Reminder Condition and the number of rats in each group are provided in Figure 2.

Given the nature of this study, it was necessary to vary the time between surgery and postoperative testing in the No-Re- minder and Reminder Conditions. The control rats were tested $24 \mathrm{~h}$ after surgery, a delay that allowed sufficient time to recover from the minor surgery and establish the type of memory representation that governed their behavior at that point. The HPC groups were lesioned at the same times but, as in similar, previous studies (e.g., Debiec et al. 2002), they were tested seven days later as that much time is required to recover from the more invasive surgery. As indicated in the Results section, the behavior of the respective groups was affected by the dominant form of memory at the time of surgery and not by the amount of time between surgery and testing.

\section{Acknowledgments}

The research was supported by grants from the Natural Sciences and Engineering Research Council to G.W. and M.M. and from the Canadian Institutes of Health Research to P.W.F. We thank Dr. Malcolm Binns for help with data analysis and Chelsea Good, Jacy Lee, and Lucy McGarry for technical assistance.

\section{References}

Alberini CM. 2007. Reconsolidation: The samsara of memory consolidation Debates Neurosci 1: 17-24.

Anagnostaras SG, Maren S, Fanselow MS. 1999. Temporally graded retrograde amnesia of contextual fear after hippocampal damage in rats: Within-subjects examination. J Neurosci 19: 1106-1114.

Biedenkapp JC, Rudy JW. 2004. Context memories and reactivation: Constraints on the reconsolidation hypothesis. Behav Neurosci 118: 956-964.

Biedenkapp JC, Rudy JW. 2007. Context preexposure prevents forgetting of a contextual fear memory: Implication for region change in brain activation patterns associated with recent and remote memory tests. Learn Mem 14: 200-203.

Bontempi B, Laurent-Demir C, Destrade C, Jaffard R. 1999. Time-dependent reorganization of brain circuitry underlying long-term memory storage. Nature 400: 671-675.

Debiec J, LeDoux JE, Nader K. 2002. Cellular and systems reconsolidation in the hippocampus. Neuron 36: 527-538.

Debiec J, Doyere V, Nader K, Ledoux JE. 2006. Directly reactivated, but not indirectly reactivated, memories undergo reconsolidation in the amygdala. Proc Natl Acad Sci 103: 3428-3433.

Dudai Y. 2004. The neurobiology of consolidations, or, how stable is the engram? Annu Rev Psychol 55: 51-86.

Dudai Y. 2006. Reconsolidation: The advantage of being refocused. Curr Opin Neurobiol 16: 174-178.

Eisenberg M, Kobilo T, Berman DE, Dudai Y. 2003. Stability of retrieved memory: Inverse correlation with trace dominance. Science 301: 11021104

Frankland PW, Bontempi B. 2005. The organization of recent and remote memories. Nat Rev Neurosci 6: 119-130.

Frankland PW, Cestari V, Filipkowski RK, McDonald RJ, Silva AJ. 1998. The dorsal hippocampus is essential for context discrimination but not for contextual conditioning. Behav Neurosci 112: 863-874.

Frankland PW, Bontempi B, Talton LE, Kaczmarek L, Silva AJ. 2004. The involvement of the anterior cingulate cortex in remote contextual fear memory. Science 304: 881-883.

Frankland PW, Ding HK, Takahashi E, Suzuki A, Kida S, Silva AJ. 2006. Stability of recent and remote contextual fear memory. Learn Mem 13: 451-457.

Jarrard LE, Meldrum BS. 1993. Selective excitotoxic pathology in the rat hippocampus. Neuropathol Appl Neurobiol 19: 381-389.

Kim JJ, Fanselow MS. 1992. Modality-specific retrograde amnesia of fear. Science 256: 675-677.

Lehmann H, Lacanilao S, Sutherland RJ. 2007. Complete or partial hippocampal damage produces equivalent retrograde amnesia for remote contextual fear memories. Eur I Neurosci 25: 1278-1286.

Maviel T, Durkin TP, Menzaghi F, Bontempi B. 2004. Sites of neocortical reorganization critical for remote spatial memory. Science 305: 96-99.

Milekic M, Alberini C. 2002. Temporally graded requirement for protein synthesis following memory reactivation. Neuron 36: $521-555$.

Morris RG, Inglis J, Ainge JA, Olverman HJ, Tulloch J, Dudai Y, Kelly PA. 2006. Memory reconsolidation: Sensitivity of spatial memory to inhibition of protein synthesis in dorsal hippocampus during encoding and retrieval. Neuron 50: 479-489.

Moscovitch M, Rosenbaum RS, Gilboa A, Addis DR, Westmacott R, Grady C, McAndrews MP, Levine B, Black S, Winocur G, et al. 2005. Functional neuroanatomy of remote episodic, semantic and spatial memory: A unified account based on multiple trace theory. J Anat 207: 35-66. 
Moscovitch M, Nadel L, Winocur G, Gilboa A, Rosenbaum SR. 2006. The cognitive neuroscience of remote episodic, semantic, and spatial memory. Curr Opin Neurobiol 16: 179-190.

Moses SN, Winocur G, Ryan JD, Moscovitch M. 2007. Environmental complexity affects contextual fear conditioning following hippocampal lesions in rats. Hippocampus 17: 333-337.

Nader K. 2003a. Memory traces unbound. Trends Neurosci 26: 65-72.

Nader K. 2003b. Neuroscience: Re-recording human memories. Nature 425: 571-572.

Nader K, Hardt O. 2009. A single standard for memory: The case for reconsolidation. Nat Rev Neurosci 10: $224-234$.

Nader K, Schafe GE, Le Doux JE. 2000. Fear memories require protein synthesis in the amygdala for reconsolidation after retrieval. Nature 406: $722-726$.

Packard M, McGaugh JL. 1992. Double dissociation of fornix and caudate nucleus lesions on acquisition of two water maze tests: Further evidence for multiple memory systems. Behav Neurosci 106: 439-446.

Paxinos G, Watson C. 1997. The rat brain in stereotaxic coordinates. Elsevier Academic Press, Amsterdam, The Netherlands.

Phillips RG, Ledoux JE. 1994. Lesions of the dorsal hippocampal formation interfere with background but not foreground contextual fear conditioning. Learn Mem 1: 34-44.

Remondes M, Schuman EM. 2004. Role for a cortical input to hippocampal area CA1 in the consolidation of a long-term memory. Nature 431: 699703.

Rosenbaum SR, Winocur G, Moscovitch M. 2001. New views on old memories: Re-evaluating the role of the hippocampal complex. Behav Brain Res 127: 183-197.

Ross RS, Eichenbaum H. 2006. Dynamics of hippocampal and cortical activation during consolidation of a nonspatial memory. J Neurosci 26: 4852-4859.

Sara SJ. 1973. Recovery from hypoxia and ECS-induced amnesia after a single exposure to training environment. Physiol Behav 10: 85-89.
Sara SJ. 2007. Retrieval and reconsolidation: Toward a neurobiology of remembering. Learn Mem 7: 73-84.

Squire LR, Alvarez P. 1995. Retrograde amnesia and memory consolidation: A neurobiological perspective. Curr Opin Neurobiol 5: 169-177.

Sutherland R, Lehmann H, Spanswick S, Sparks F, Melvin N. 2006. Growth points in research on memory and hippocampus. Cdn J Exp Psy 60: 166 174 .

Sutherland RJ, O'Brien J, Lehmann H. 2008. Absence of systems consolidation of fear memories after dorsal, ventral, or complete hippocampal damage. Hippocampus 18: 710-718.

Suzuki A, Josselyn SA, Frankland PW, Masushige S, Silva AJ, Kida S. 2004. Memory reconsolidation and extinction have distinct temporal and biochemical signatures. J Neurosci 24: 4787-4795.

Wang SH, Teixeira CM, Wheeler AL, Frankland PW. 2009. The precision of remote context memories does not require the hippocampus. Nat Neurosci 12: 253-255.

Wiltgen BJ, Silva AJ. 2007a. Memory for context becomes less specific with time. Learn Mem 14: 313-317.

Wiltgen BJ, Silva AJ. 2007b. Neural circuits mediating the retrieval of specific and generalized context fear. Poster presented at the Annual Meeting of the Society for Neuroscience, San Diego, CA.

Winocur G, Moscovitch M, Fogel S, Rosenbaum RS, Sekeres M. 2005. Preserved spatial memory after hippocampal lesions: Effects of extensive experience in a complex environment. Nat Neurosci 8: 273-275.

Winocur G, Wojtowicz JM, Sekeres M, Snyder JS, Wang S. 2006. Inhibition of neurogenesis interferes with hippocampus-dependent memory function. Hippocampus 16: 296-304.

Winocur G, Moscovitch M, Sekeres M. 2007. Memory consolidation or transformation: context manipulation and hippocampal representations of memory. Nat Neurosci 10: 555-557.

Received April 6, 2009; accepted in revised form August 25, 2009. 


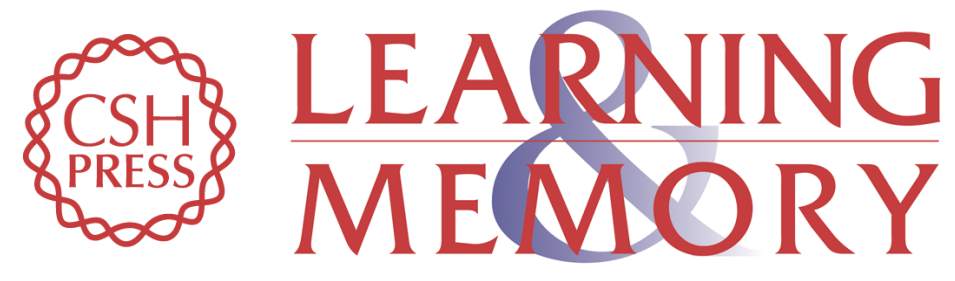

\section{Changes in context-specificity during memory reconsolidation: Selective effects of hippocampal lesions}

Gordon Winocur, Paul W. Frankland, Melanie Sekeres, et al.

Learn. Mem. 2009, 16:

Access the most recent version at doi:10.1101//m.1447209

Supplemental
Material http://learnmem.cshlp.org/content/suppl/2009/11/24/16.11.722.DC1

References This article cites 43 articles, 12 of which can be accessed free at: http://learnmem.cshlp.org/content/16/11/722.full.html\#ref-list-1

License

Email Alerting Receive free email alerts when new articles cite this article - sign up in the box at the Service top right corner of the article or click here. 